\title{
Thought translation, tennis and Turing tests in the vegetative state
}

\author{
John F. Stins • Steven Laureys
}

Published online: 10 March 2009

(C) The Author(s) 2009. This article is published with open access at Springerlink.com

\begin{abstract}
Brain damage can cause massive changes in consciousness levels. From a clinical and ethical point of view it is desirable to assess the level of residual consciousness in unresponsive patients. However, no direct measure of consciousness exists, so we run into the philosophical problem of other minds. Neurologists often make implicit use of a Turing test-like procedure in an attempt to gain access to damaged minds, by monitoring and interpreting neurobehavioral responses. New brain imaging techniques are now being developed that permit communication with unresponsive patients, using their brain signals as carriers of messages relating to their mental states.
\end{abstract}

Keywords Consciousness $\cdot$ Turing test $\cdot$ Vegetative state $\cdot$ Other minds

\section{Introduction}

Scientists and laymen seem to agree on at least three important aspects of consciousness. First, only living animals can have consciousness, whereas other forms of life such as plants and non-living objects such as rocks and chairs cannot. With the possible exception of a computer of sufficient complexity so that consciousness may emerge from it (e.g., Minsky 1982), it is generally agreed that objects and artifacts lack consciousness. Second, the contents of our consciousness can be shared with other human beings through acts of communication, which allows us to exchange thoughts, feelings, and ideas with others. The medium through which the contents of our

\footnotetext{
J. F. Stins $(\bowtie)$

Research Institute MOVE, Faculty of Human Movement Sciences, VU University Amsterdam, van der Boechorststraat 9, 1081 BT Amsterdam, The Netherlands

e-mail: j.stins@fbw.vu.nl

S. Laureys

Coma Science Group, Cyclotron Research Center and Neurology Department, University of Liège, Sart Tilman B30, 4000 Liège, Belgium

e-mail: steven.laureys@ulg.ac.be
} 
consciousness are exchanged can involve verbal and non-verbal communication such as speech, sign language, morse code, facial expressions, works of art, etc. Third, the fact that an agent possesses consciousness in part shapes our moral attitude toward it (Knobe and Prinz 2008). For example, most of us now believe that dogs possess some sort of consciousness, and we therefore feel a moral obligation to feed it, play with it, and minimize suffering when the animal is hurt. If, on the other hand, we are dealing with a robot dog, we will not ascribe consciousness to it and we will belief the dog can experience neither joy nor pain. In this case, a broken 'leg' will at most constitute financial suffering on behalf of the owner. However, in pathological instances involving severe brain damage we can no longer rely on our intuitions concerning consciousness in others. More specifically, in some cases brain damage may result in a complete and permanent cessation of volitional bodily movements, and hence a loss of expressive capabilities. As outside observers who are confronted with such patients we can thus no longer rely on bodily (including verbal) cues for communication. Since the loss of expressive capabilities implies incapacity to report on mental states, we may be tempted to conclude that the patient is no longer conscious. However it could also be the case that the patient is consciously aware of self and surroundings, but that conventional approaches for communication are insufficient. The core question then becomes: what entitles us to ascribe consciousness (or lack thereof) to brain damaged patients who have lost all capabilities to communicate? The aim of this paper is to expose some of the philosophical and practical issues surrounding our attempts to establish consciousness in non-communicative patients following severe acute brain damage.

\section{Damaged brain, damaged mind? The other minds problem in neurology}

A frequent outcome of traumatic brain injury, especially damage to the brainstem or midbrain, is the state of coma, which is characterized by a complete loss of consciousness and wakefulness. More specifically, individuals in a comatose brain state are no longer conscious of themselves or their surroundings, and they are unable to produce willed movements. But coma is hardly ever a permanent condition, and after a while the patient may progress into one of multiple clinical states resembling coma (e.g., Laureys 2007; Laureys and Boly 2008) that pose puzzling questions for researchers and relatives. One of several possible outcomes of coma is that the individual regains consciousness, but s/he remains completely paralyzed. This condition is known as locked-in-syndrome (LIS; e.g., Laureys et al. 2005). The incapacity to generate bodily movements prohibits communication through conventional means, and to an outside observer the near-complete absence of signs of responsiveness creates the impression of consciousness being absent, resembling deep sleep or general anesthesia. Yet, individuals in LIS often retain the ability to move certain ocular muscles, and they may learn to express themselves through movements of an eye lid. Following a brainstem stroke in 1995, the French journalist Jean-Dominique Bauby was completely paralyzed. Amazingly, through an immense effort, he succeeded in writing about his memoires (The Diving Bell and the Butterfly) by dictating a letter at a time with his left eye lid.

Another outcome of coma may be the vegetative state (VS) which, after prolonged duration (i.e., one month), is labeled the persistent vegetative state 
(PVS). Contrary to coma, individuals in VS have intact sleep-wake cycles and they are able to breathe without support. In addition, individuals in VS may on occasion produce facial movements, generate sounds such as cries and moans, and show random eye movements. Yet, the medical consensus is that these patients completely lack awareness of their surroundings, and hence of themselves. The occasionally observed bodily movements are viewed as motor reflexes that emerge at irregular arbitrary intervals. Yet, families and friends of the patient sometimes have the compelling impression that certain movements (especially movements of the eye, or grasping movements) are volitional and that the patient is somehow aware of their presence, despite the clinician's assertion that these movements are purely reflexive and automatic (cf. Laureys 2005a). Our subjective interpretation of these movements thus determines our assessment of the level of consciousness within such patients (e.g., Owen et al. 2009). This example demonstrates that the tendency to ascribe consciousness and intentionality to non-conscious agents can be very strong. As another example of this tendency, if we are in the vicinity of a robot that follows our movements as we walk up and down a room by rotating its artificial head and eyes, we get the creepy feeling that we are being watched, even though we know at an abstract level that we are just dealing with a machine. Thus, if we witness an entity producing a set of coordinated movements that resemble purposeful movement, we are strongly inclined to evaluate these movements as originating from a conscious intentional agent.

In individual cases it may be difficult to determine whether a post-comatose patient is locked-in or resides in VS (e.g., Kobylarz and Schiff 2004). Yet, the purported presence or absence of consciousness strongly determines our moral decisions toward the brain damaged individual. In the case of LIS, where we are told the person is conscious, yet she has no conventional way of expressing her thoughts, we may decide to sit at the bed side and tell stories to her and try to comfort her. If, on the other hand, physical examination reveals that consciousness is lost and will never be regained, as is often the case in VS, then this has serious repercussions for future treatment. An extreme outcome could be that we decide to withhold artificial feeding and hydration, as was done in the case of Terri Schiavo in 2005 in the United States and more recently in the case of Eluana Englaro in Italy. Such a drastic decision should of course not be based exclusively on considerations regarding consciousness, but should also be informed by personal moral values and by cultural and legal practices in the community (Laureys 2005b).

A clinician trying to assess the level of consciousness in unresponsive patients as part of medical examination is facing a number of practical problems. For example, bodily signs of consciousness may be feeble and barely perceptible. Furthermore, repeated examinations are required before the diagnosis is reached; for example, the patient may have been asleep at the time of testing, which of course should not be interpreted as 'unconscious'. But underneath these practical considerations there lurks a deeper philosophical problem that is reminiscent of the 'other minds problem'. The epistemologic challenge facing us is that we have no direct knowledge of the mental states of others, and we typically rely on linguistic utterances and gestures as a window into the contents of the minds of others.

But given that others have bodies and sensory organs that are very similar to ours, we have very good reason to suppose that others also experience the world in a 
similar fashion. For example, if I feel an itch then I have a strong disposition to scratch. If someone else observes me scratching, then s/he has very good reason to assume that I indeed experience an itch. One solution to the other minds problem states that itching and scratching are causally related, i.e., not merely in a contingent fashion (e.g., Aune 1963). However, as a result of brain damage the link (causal or otherwise) between mental state and behavioral manifestation may have been altered or broken, and we can no longer rely on conventional methods to gain access to the minds of such patients (see below). We here assert that the problem of other minds lurks beneath the surface with respect to the examination of patients in the vegetative state. Our clinical method for determining whether a patient is conscious is by eliciting a predicted response to an external prompt or command. As was pointed out by Kurthen et al. (1991), clinicians tend to adopt an essentially behaviorist approach to impaired consciousness. That is, they are trying to elicit some sort of response of the patient in the form of spoken words or a non-verbal signal, such as an oriented response to an applied noxious stimulation, the reproducible tracking of an object moved in front of the patient's eyes or (most convincingly) a movement as simple as a blink of an eye in direct response to a simple command. It is this motor responsiveness and this motor responsiveness only that allows others to asses the residual level of mental functioning. Indeed, after careful observation of feeble bodily signals, (e.g., barely perceptible eye movements) the clinician may conclude that the most parsimonious explanation of these movements is that they are under voluntary control, and that hence the patient is conscious. In short, our ability to know unequivocally that another being is consciously aware is determined by the patient's ability to communicate that awareness through a recognized contingent motor response.

The conceptual difficulties surrounding our inherent limitations in trying to assess consciousness in unresponsive patients have some unexpected parallels in the seemingly unrelated field of artificial intelligence (A.I.), and more specifically the A.I. debate concerning whether machines can think (Stins 2008). This debate was more or less sparked off by a landmark paper by the great mathematician Alan Turing (1950). Turing was interested in the possibility of 'intelligent' or 'thinking' machines. He acknowledged the difficulties surrounding defining the concept of intelligence and he therefore came up with an operational measure of machine intelligence in the form of a test: if a human interrogator engages in a conversation with the computer using a teleprinter device, and the interrogator is unable to decide whether s/he is conversing with a computer or with another human being, then according to Turing the machine must be intelligent. In other words, if following a question-and-answer session a cleverly programmed computer tricks us into believing we are communicating with a human, then we must at some stage conclude we are dealing with an intelligent agent. According to Turing, it would be perfectly reasonable to call the computer 'intelligent', because we judge other people's intelligence in exactly the same way, that is, from external observation. This thought experiment is now known as the "Turing Test", and has reached an almost iconic status in the A.I. literature, and figures prominently in the discussion surrounding the so-called Strong A.I. claim. Note that in more modern versions of the Turing Test "intelligence" has been replaced by "consciousness". Although these are different concepts, they both refer to a unique human mental capability. 
The crucial point raised by Turing in his paper is that there exist inherent limitations in our ability to assess whether another agent is truly conscious, by means of a communication protocol. To make matters worse, the same problem exists when we are dealing face-to-face with other human beings; at best we can only infer the presence of consciousness in others. As Turing (1950) expressed it in a succinct British fashion: "it is usual to have the polite convention that everyone thinks." There is indeed a close connection between the modern problem whether artificial devices have minds, and the older philosophical problem of how we know for certain that others have a mind, based on behavioral evidence (e.g., Harnad 1991).

As argued by Stins (2008), neurologists and scientists who are trying to establish the level of consciousness in unresponsive brain damaged patients implicitly adopt a Turing-test- like procedure in their efforts to expose mental states. Basically, they are looking for signs of contingency and communication in the patient; either in the form of simple command following, verbal report, systematic blinks of an eye lid, oriented response to pain or 'itching', eye-tracking etc., and these signs are interpreted either as 'automatic' or 'volitional'. However, when dealing with VS behavior, bodily movements seem to be exclusively of an automatic reflex-like nature. But does the absence of volitional movements necessarily imply an absence of consciousness? A number of recent studies took another approach and looked directly at patterns of brain activation so as to gain insight into the extent of spared neural and mental functioning in these patients.

\section{Neuroimaging of the damaged brain: Let's try some mind decoding}

A small number of functional neuroimaging experiments tried to elucidate whether in VS some degree of processing still existed of noxious ('painful') stimuli (e.g., Kassubek et al. 2003; Laureys et al. 2002), whether complex linguistic material could be processed (e.g., Owen 2008), or whether subjects responded to hearing their own name (e.g., Laureys et al. 2007; Perrin et al. 2006; Di et al. 2007). Basically, in these experiments neural activation in response to different stimuli is assessed using standard neuroimaging techniques, such as the electro-encephalogram (EEG), positron emission tomography (PET) or functional magnetic resonance imaging (fMRI). The pattern of activation recorded in groups of VS patients is compared to that of controls and this comparison allows the researcher to assess the extent of spared cognitive processing in VS. One of the remarkable outcomes of these studies is that neural processing in VS can be very similar to processing in conscious control subjects, which suggests some degree of intact cognitive functioning. Yet, in contrast to controls, neural activity in VS appears to remain 'isolated' in the nervous system, that is, neural activity is not integrated with other neural patterns within cortical association areas. Thus, despite the absence of awareness discrete islands of cognitive functioning may still exist (e.g., Owen 2008). According to one interpretation (Laureys 2005a) VS can be considered a disconnection syndrome whereby the functional and effective connectivity of the brain (notably cortico-cortical and cortico- thalamo-cortical connections) has been compromised.

Despite the sometimes striking similarities between the healthy brain and the VS brain, the consensus is that these brain patterns cannot be used as evidence of 
consciousness in these brains. For one, the neural correlate of consciousness has not yet been identified. As a result, "even a near-to-normal activation in response to passive sensory stimulation cannot be considered as a proof of the presence of awareness in these [VS] patients." (Boly et al. 2007; p. 980).

A recent study by Owen et al. (2006) added a whole new twist to the debate by adopting a novel approach (Boly et al. 2007) to the traditional neuroimaging studies. Owen et al. (2006) performed an fMRI study using a single female patient who had been in VS for 5 months, and they asked the woman to engage in one of several mental activities while she was being scanned. The woman received auditory instructions to either imagine herself playing tennis (a motor task), or to imagine herself walking through her house (a spatial navigation task). Note that this woman was completely unresponsive, and the researchers had thus no way of assessing whether she did as requested. Upon analysis of the BOLD signal the researchers found something amazing: the differential pattern of brain activation in both conditions was indistinguishable from the neural signatures obtained with (conscious) control subjects. When asked to imagine tennis, both the patient and the controls showed activation in the supplementary motor area. During the spatial navigation task, in contrast, robust activation was found in other areas, such as the parahippocampal gyrus and the parietal cortex. Based on these results Owen et al. (2006) drew the far reaching conclusion that this woman must have been conscious, and that she demonstrated her conscious awareness via her brain activation. Owen and Coleman (2008) phrased their conclusion as follows: "Despite the fact that [this woman] fulfilled all of the clinical criteria for a diagnosis of vegetative state, the patient retained the ability to understand spoken commands and respond to them through her brain activity, confirming beyond any doubt that she was consciously aware of herself and her surroundings." (p. 238; see also Owen 2008). Crucially, this radical conclusion was reached by giving a whole new interpretation to the fMRI signal: it was not only seen as a signature of mental activity; the signal itself was treated as a behavioral response. More specifically, this woman appeared to demonstrate the ability to understand instructions and to willfully comply with the request of the experimenters; not by means of any overt bodily action, but by means of modulation of brain responses. The fact that this woman had managed to convince others that she was conscious is tantamount to saying that she had passed the Turing test (Stins 2008; see also Naccache 2006). As an aside, it should be noted that analysis and interpretation of fMRI signals in general requires extensive pre-processing, which may for now limit the possibilities to use the above procedure for real-time communication.

The suggestion to treat brain signals originating from the brain of an unresponsive patient as a behavioral response is an original one, yet it is not completely new. Kurthen et al. (1991) already considered the possibility to treat electrophysiological events as instances of behavior in the case where the possibility to perform any sort of voluntary motor function is lost, despite preserved consciousness, which they called total locked-in syndrome (TLIS). Kurthen et al. (1991) developed a philosophical line of reasoning to expose the limits of a behaviorist account of the other minds problem in general, and they used the case of TLIS as an example to highlight the problems faced by neurologists and clinicians who implicitly adopt a behaviorist approach to assessing consciousness in brain damaged patients. 
According to Kurthen et al. (1991) the problem of other minds cannot be solved by stretching the notion of 'behavior' to include neural patterns, because we are still faced with the problem of how we can utilize extrinsic signs of consciousness (behavioral or neurophysiological) to determine the intrinsic aspects of consciousness (phenomenality and subjectivity). The limits of a behaviorist epistemology of other minds could, so it was argued, be surpassed by adopting a solution to the problem that is essentially based on Wittgenstein's ideas surrounding 'other minds'. Although this idea has some clear philosophical merits, Kurthen et al. (1991) also acknowledged the practical issues at stake and they concluded that for the time being it is probably best "to treat potential TLIS patients as if they are conscious." (p. 77; emphasis in the original).

\section{The way ahead}

How should this line of research proceed? Given the growing availability and reliability of brain scanning techniques it will become increasingly feasible to explore whole new ways of communicating with unresponsive patients (e.g., Owen 2008). The innovative methodology proposed by Boly et al. (2007) convincingly demonstrated that this practice of 'rudimentary mind reading' can be applied to individual cases, as opposed to the often used group-based analysis techniques (Owen et al. 2006). It has recently been proposed to use brain signals themselves as a medium of communication with VS patients and patients in the minimally conscious state (MCS; Schnakers et al. 2008) and with LIS patients (Egeth 2008; cf. Kurthen et al. 1991). The proposal of Egeth (2008) was based on the established reliability of the so-called P300 or mismatch negativity (MMN; Egeth 2008) ; both P300 and MMN are clear peaks in the EEG signal following an attended or unexpected auditory stimulus. Crucially, which of two types of auditory stimuli counts as expected or as unexpected depends on what stimuli we choose to attend to. As a result, consciously attending to one or the other stream can yield a clearly identifiable binary pattern in the EEG signal, which can than be mapped onto a 'yes' or a 'no' response. Based on this idea, Egeth (2008) and Laureys and Boly (2008) argued that we can in principle, and probably also in practice, create a braincomputer interface (BCI) that allows us to engage in a simple form of conversation with locked-in subjects, simply by interpreting brain signals in a well- defined fashion (also see Vanhaudenhuyse et al. 2007).

Recently, Kübler (2009) wrote an excellent review on BCI's, and their application for the examination of cognitive processes in patients with disorders of consciousness. Of course, BCI's hold the promise of communication with patients who have lost all control over their motor periphery. As pointed out by Kübler (2009), a distinction should be drawn between so-called passive stimulation paradigms and volitional paradigms. The passive stimulation paradigm involves recording of brain activity while the patient is presented with sensory stimulation, usually of a verbal nature. Analysis of the frequency contents in the EEG or the occurrence of eventrelated peaks in the signal allows the researcher to establish the extent of spared cognitive functioning, e.g., whether the brain responds to semantic anomalies in the stimulus pattern. The volitional paradigm, in contrast, is designed such that 
successful task performance requires understanding of instructions, the will to perform as required, and the capability to perform as requested (Kübler 2009). An example of the successful application of this paradigm is of course the mental imagery study performed by Owen et al. (2006). The fact that their VS patient understood the instructions and complied with the requests is compelling evidence for the presence of consciousness. Note that the passive stimulation paradigm can be used to assess the extent of spared cognitive functioning, but not to assess the level of consciousness in unresponsive patients.

We see the Owen et al. (2006) study and Laureys and Boly's (2008) proposal as important first steps in the development of an interface to gain access to minds that cannot rely on verbal or bodily signs to make themselves known. In our view, if we want to engage in a Turing-like test procedure with unresponsive brain damaged patients using only their brain signals as carrier of information, at least four conditions have to be met. First, the brain signal must have a high reliability, i.e., a high signal-to-noise ratio. Otherwise, we may need many repetitions, which may undermine the validity of the procedure. Second, the brain signals must be contingent on external prompts or command -hence reflecting the patient's voluntary control. This is not as simple as it seems, given that much of our brain activation is automatic and goes on without our conscious control (for example, we cannot decide not to understand a word spoken to us, and our language centers will switch on automatically). Brain signals that cannot be controlled are thus of little use, and will only reveal islands of cognition. Third, for practical purposes it is desirable that the BCI can be used with as little training as possible. As an example, healthy subjects are in general capable of moving a mouse cursor by modulating their brain activity, and thus play video games or surf the internet. However, this requires extensive practice, using principles of operant conditioning and by providing neurofeedback, and it is unclear whether this skill can be mastered by brain damaged patients within a reasonable amount of time. Moreover, prior to "hooking up" a damaged brain to a $\mathrm{BCI}$ it is desirable to know the level of mental functioning in the first place which, as argued in this paper, is far from straightforward. Fourth, the brain signals need to be properly interpreted, e.g., pattern A signals 'yes', and pattern B signals 'no'. If we manage to create some sort of Rosetta Stone that allows us to translate overt brain patterns (or other bodily signals) to private thoughts there can in principle be communication. Some innovative techniques are now being developed that allow registration of binary (yes-no) responses from non-communicative patients, e.g., by recording changes in salivary $\mathrm{pH}$ (Vanhaudenhuyse et al. 2007; Wilhelm et al. 2007) or changes in levels of subclinical muscle activation (Bruno et al. 2008; Lakerveld et al. 2008) in response to spoken commands. If we want to open up the possibility for patients who are unresponsive yet consciously aware to pass the Turing test then we should adopt voluntary brain-controlled non-motor signals in our repertoire of recognized behavioral responses.

As a final note, in this paper we have treated the body as a potential vehicle for expression, and we have shown that the incapacity to generate voluntary bodily movements poses practical and philosophical problems for our assessment of consciousness in brain damaged individuals. Of course, lack of movement control has also serious repercussions for the mind of the affected individual himself or herself. For example, as was pointed out by O'Regan et al. (2005), having a sensory 
experience is fundamentally linked with implicit knowledge of how to modulate that sensation, e.g. by engaging in an act of exploration. The fact that the brain damaged individuals discussed in this paper have now become mere passive receivers of stimuli as a result of their pathology undoubtedly shapes their minds. The challenge remains how the contents of the private mind in these patients can be made public.

Open Access This article is distributed under the terms of the Creative Commons Attribution Noncommercial License which permits any noncommercial use, distribution, and reproduction in any medium, provided the original author(s) and source are credited.

\section{References}

Aune, B. (1963). Feelings, moods and introspection. Mind, 62, 187-208.

Boly, M., Coleman, M. R., Davis, M. H., Hampshire, A., Bor, D., Moonen, G., et al. (2007). When thoughts become action: an fMRI paradigm to study volitional brain activity in non-communicative brain injured patients. NeuroImage, 36, 979-992.

Bruno, M., Bernheim J. L,. Schnakers C., \& Laureys, S. (2008). Locked-in: don't judge a book by its cover. Journal of Neurology, Neurosurgery and Psychiatry, 79, 3.

Di, H. B., Yu, S. M., Weng, X. C., Laureys, S., Yu, D., Li, J. Q., et al. (2007). Cerebral response to patient's own name in the vegetative and minimally conscious states. Neurology, 68, 895-899.

Egeth, M. (2008). A “Turing Test” and BCI for locked-in children and adults. Medical Hypotheses, 70, 1067.

Harnad, S. (1991). Other bodies, other minds: a machine incarnation of an old philosophical problem. Minds and Machines, 1, 43-54.

Kassubek, J., Juengling, F. D., Els, T., Spreer, J., Herpers, M., Krause, T., et al. (2003). Activation of a residual cortical network during painful stimulation in long-term postanoxic vegetative state: a ${ }^{15} \mathrm{O}-$ $\mathrm{H}_{2} \mathrm{O}$ PET study. Journal of the Neurological Sciences, 212, 85-91.

Knobe, J., \& Prinz, J. (2008). Intuitions about consciousness: experimental studies. Phenomenology and the Cognitive Sciences, 7, 67-83.

Kobylarz, E. J., \& Schiff, N. D. (2004). Functional imaging of severely brain-injured patients. Archives of Neurology, 61, 1357-1360.

Kübler, A. (2009). Brain-computer interfaces for communication in paralysed patients and implications for disorders of consciousness. In S. Laureys \& G. Tononi (Eds.), The Neurology of Consciousness (pp. 217-233). Elsevier.

Kurthen, M., Moskopp, D., Linke, D. B., \& Reuter, B. M. (1991). The locked-in syndrome and the behaviorist epistemology of other minds. Theoretical Medicine, 12, 69-79.

Lakerveld, J., Kotchoubey, B., \& Kübler, A. (2008). Cognitive function in patients with late stage amyotrophic lateral sclerosis. Journal of Neurology, Neurosurgery and Psychiatry, 79, 25-29.

Laureys, S. (2005a). The neural correlate of (un) awareness: lessons from the vegetative state. Trends in Cognitive Science, 9, 556-559.

Laureys, S. (2005b). Death, unconsciousness and the brain. Nature Reviews Neuroscience, 6, 899-909.

Laureys, S. (2007). Eyes open, brain shut: the vegetative state. Scientific American, 4, 32-37.

Laureys, S., \& Boly, M. (2008). The changing spectrum of coma. Nature Clinical Practice Neurology, 4, 544-546.

Laureys, S., Faymonville, M. E., Peigneux, P., Damas, P., Lambermont, B., Del Fiore, G., et al. (2002). Cortical processing of noxious somatosensory stimuli in the persistent vegetative state. NeuroImage, 17, 732-741.

Laureys, S., Pellas, F., van Eeckhout, P., Ghorbel, S., Schnakers, C., Perrin, F., et al. (2005). The locked-in syndrome: what is it like to be conscious but paralyzed and voiceless? Progress in Brain Research, 150, 495-511.

Laureys, S., Perrin, F., \& Brédart, S. (2007). Self-consciousness in non-communicative patients. Consciousness and Cognition, 16, 722-741.

Minsky, M. (1982). Why people think computers can't. AI Magazine, 3, 3-15.

Naccache, L. (2006). Is she conscious? Science, 313, 1395-1396. 
O’Regan, K., Myin, E., \& Noë, A. (2005). Skill, corporality and alerting capacity in an account of sensory consciousness. Progress in Brain Research, 150, 55-68.

Owen, A. M. (2008). Disorders of consciousness. Annals of the New York Academy of Sciences, 1124, 225-238.

Owen, A. M., \& Coleman, M. R. (2008). Functional neuroimaging of the vegetative state. Nature Reviews Neuroscience, 9, 235-243.

Owen, A. M., Coleman, M. R., Boly, M., Davis, M. H., Laureys, S., \& Pickard, J. D. (2006). Detecting awareness in the vegetative state. Science, 313, 1402.

Owen, A.M., Schiff, N.D., \& Laureys, S. (2009). The assessment of conscious awareness in the vegetative state. In S. Laureys \& G. Tononi (Eds.), The Neurology of Consciousness (pp. 163-172). Elsevier.

Perrin, F., Schnakers, C., Schabus, M., Degueldre, C., Goldman, S., Brédart, S., et al. (2006). Brain response to one's own name in vegetative state, minimally conscious state, and locked-in syndrome. Archives of Neurology, 63, 562-569.

Schnakers, C., Perrin, F., Schabus, M., Majerus, S., Ledoux, D., Damas, P., et al. (2008). Voluntary brain processing in disorders of consciousness. Neurology, 71, 1614-1620.

Stins, J. F. (2008). Establishing consciousness in non-communicative patients: a modern-day version of the Turing test. Consciousness and Cognition. doi:10.1016/j.concog.2007.12.005.

Turing, A. M. (1950). Computing machinery and intelligence. Mind, 59, 433-460.

Vanhaudenhuyse, A., Bruno, M.-A., Brédart, S., Plenevaux, A., \& Laureys, S. (2007). The challenge of disentangling reportability and phenomenal consciousness in post-comatose states. Behavioral and Brain Sciences, 5-6, 529-530.

Wilhelm, B., Jordan, M., \& Birbaumer, N. (2007). Communication in locked-in syndrome: effects of imagery on salivary pH. Neurology, 67, 534-535. 